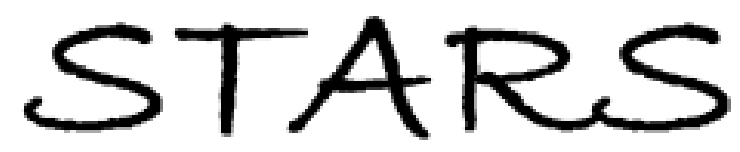

University of Central Florida

STARS

$1-1-2012$

\title{
Unstaggered-staggered solitons in two-component discrete nonlinear Schrodinger lattices
}

\author{
Boris A. Malomed \\ D. J. Kaup \\ University of Central Florida \\ Robert A. Van Gorder \\ University of Central Florida
}

Find similar works at: https://stars.library.ucf.edu/facultybib2010

University of Central Florida Libraries http://library.ucf.edu

This Article is brought to you for free and open access by the Faculty Bibliography at STARS. It has been accepted for inclusion in Faculty Bibliography 2010 s by an authorized administrator of STARS. For more information, please contactSTARS@ucf.edu.

\section{Recommended Citation}

Malomed, Boris A.; Kaup, D. J.; and Van Gorder, Robert A., "Unstaggered-staggered solitons in twocomponent discrete nonlinear Schrodinger lattices" (2012). Faculty Bibliography 2010s. 2994.

https://stars.library.ucf.edu/facultybib2010/2994

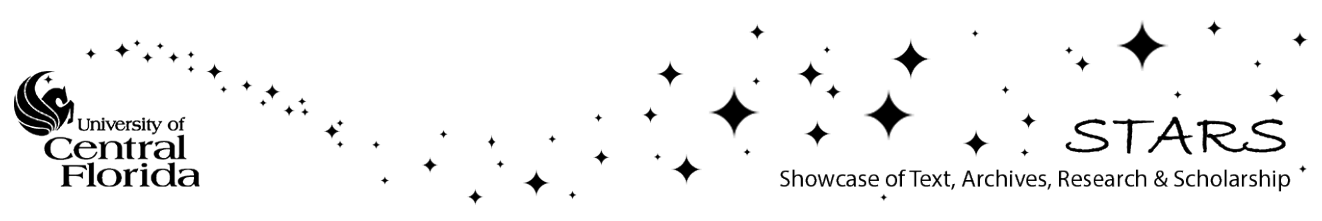




\title{
Unstaggered-staggered solitons in two-component discrete nonlinear Schrödinger lattices
}

\author{
Boris A. Malomed, ${ }^{1}$ D. J. Kaup, ${ }^{2}$ and Robert A. Van Gorder ${ }^{3}$ \\ ${ }^{1}$ Department of Physical Electronics, School of Electrical Engineering, Faculty of Engineering, Tel Aviv University, Tel Aviv 69978, Israel \\ ${ }^{2}$ Department of Mathematics \& Institute for Simulation and Training, University of Central Florida, Orlando, Florida 32816-1364, USA \\ ${ }^{3}$ Department of Mathematics, University of Central Florida, Orlando, Florida 32816-1364, USA
}

(Received 18 December 2011; published 16 February 2012)

\begin{abstract}
We present stable bright solitons built of coupled unstaggered and staggered components in a symmetric system of two discrete nonlinear Schrödinger equations with the attractive self-phase-modulation nonlinearity, coupled by the repulsive cross-phase-modulation interaction. These mixed modes are of a "symbiotic" type, as each component in isolation may only carry ordinary unstaggered solitons. The results are obtained in an analytical form, using the variational and Thomas-Fermi approximations (VA and TFA), and the generalized Vakhitov-Kolokolov (VK) criterion for the evaluation of the stability. The analytical predictions are verified against numerical results. Almost all the symbiotic solitons are predicted by the VA quite accurately and are stable. Close to a boundary of the existence region of the solitons (which may feature several connected branches), there are broad solitons which are not well approximated by the VA and are unstable.
\end{abstract}

DOI: 10.1103/PhysRevE.85.026604 PACS number(s): 05.45.Yv, 42.65.Tg, 63.20.Ry, 03.75.Lm

\section{INTRODUCTION}

Discrete nonlinear Schrödinger (DNLS) equations constitute a class of lattice models which comprise diverse physical settings [1]. A straightforward realization of the DNLS equations in arrays of evanescently coupled optical waveguides was first proposed in Ref. [2] and later demonstrated experimentally in a set of parallel semiconductor waveguides [3]. Multicore nonlinear waveguiding systems have also been created in the form of optically written virtual lattices in photorefractive materials [4] and as permanent structures written by laser pulses in bulk silica [5]. A thorough review of the nonlinear discrete optics, developed experimentally and theoretically in these and allied media, was given in Ref. [6]. The DNLS equations find another important application in modeling the mean-field dynamics of Bose-Einstein condensates (BECs) loaded into deep optical-lattice potentials. In this case, it was demonstrated experimentally [7] and theoretically [8] that the periodic potential effectively splits the condensate into a set of droplets trapped in local potential wells, which are linearly coupled by tunneling of atoms across the separating potential barriers, DNLS equations being natural models for such quasidiscrete systems.

Two fundamental types of discrete solitons supported by the DNLS equations with the self-repulsive and self-attractive on-site nonlinearity are localized modes of staggered and unstaggered types, respectively, i.e., ones with opposite signs of the lattice field at adjacent sites, or without the sign alternation [1]. In the continuum limit, the unstaggered solitons correspond to regular ones, residing in the semi-infinite gap of the continual NLS equation, while the staggered solitons may be considered as counterparts of gap solitons, which exist in finite band gaps of the spectrum induced by a periodic potential, in the case of the self-defocusing nonlinearity [10].

A natural generalization, which also finds many applications to optics and BEC, is represented by systems of coupled DNLS equations. In optics, the system models the co-propagation of two waves carried by different polarizations or wavelengths in the same waveguiding array, while in BEC the coupled equations describe a mixture of two condensates, which may represent either different hyperfine states of the same atomic species, or two different kinds of atoms [9]. Normally, two-component discrete solitons in such systems feature one type of the intrinsic structure, unstaggered or staggered, in both components, because the signs of the selfphase-modulation (SPM) nonlinearity acting on each component, and of the cross-phase-modulation (XPM) nonlinearity which couples them, are the same [1]. The objective of the present work is to introduce two-component discrete solitons of the mixed type, built as combinations of unstaggered and staggered components. Previously, single-component surface modes of a mixed unstaggered-staggered type were studied at an interface between two different lattices [11], but, to the best of our knowledge, two-component mixed solitons have not been reported before. On the other hand, in bimodal continual systems with the periodic potential acting on both components, solitons of a semigap type, which may be considered as continuous counterparts of the discrete ones introduced in the present work, were studied in Ref. [12]. They are composed of an ordinary soliton in one component and a gap soliton in the other. The semigap solitons are somewhat similar to the earlier studied intergap solitons, that were built as bound states of two components represented by solitons belonging to two different finite band gaps (the first and second ones) [13].

The paper is organized as follows. The model is formulated in Sec. II. Approximate analytical results are presented in Sec. III. These results, based chiefly on the variational approximation (VA), demonstrate that the mixed unstaggeredstaggered solitons are possible in the symmetric system of DNLS equations when the XPM interaction between the two components is repulsive, on the contrary to the self-attractive SPM nonlinearity. The situation with the opposite signs of the SPM and XPM terms seems exotic in optics, but it is quite possible in BEC, where the sign of the interactions may be readily switched by means of the Feshbach resonance (see, e.g., Ref. [14]). In the case of strong difference between masses of the two components, another analytical solution is obtained, based on the Thomas-Fermi approximation (TFA). Numerical results, which allow us to outline existence regions 
of fundamental (single-peak) solitons combining the unstaggered and staggered components, and identify their stability (almost all the solitons are stable), are summarized in Sec. IV. Analytical results for the stability are reported too, based on the Vakhitov-Kolokolov (VK) criterion for the two-component system. The numerical results corroborate the predictions of the VA quite well; in particular, it is confirmed that the mixed unstaggered-staggered solitons exist only in the case of the repulsive XPM, while the SPM is self-attractive in both components. The paper is concluded by Sec. V.

\section{THE MODEL}

The underlying system of the DNLS equations for lattice fields $\phi_{n}$ and $\psi_{n}$ is

$$
\begin{aligned}
& i \frac{d}{d t} \phi_{n}=-\frac{1}{2}\left(\phi_{n+1}+\phi_{n-1}-2 \phi_{n}\right)-\left(\left|\phi_{n}\right|^{2}+\beta\left|\psi_{n}\right|^{2}\right) \phi_{n}, \\
& i \frac{d}{d t} \psi_{n}=-\frac{1}{2 m}\left(\psi_{n+1}+\psi_{n-1}-2 \psi_{n}\right)-\left(\left|\psi_{n}\right|^{2}+\beta\left|\phi_{n}\right|^{2}\right) \psi_{n},
\end{aligned}
$$

where $t$ is time in the case the BEC mixture or the propagation distance in the array of optical waveguides, $m$ is the relative atomic mass of the two species in the case of BEC or the inverse ratio of the intersite coupling constants in the waveguide array, and $\beta$ is the relative coefficient of the XPM coupling between the fields, assuming that the coefficients of the self-attractive SPM nonlinearity for both fields are scaled to be 1 . It should be mentioned that the model based on Eqs. (1) is not the most general one, as, rescaling both fields to make their SPM coefficients equal to 1 , one can make the XPM interaction asymmetric, with two different coefficients in Eqs. (1a) and (1b), $\beta_{\phi} \neq \beta_{\psi}$. Nevertheless, quite generic results concerning the discrete solitons can be obtained within the framework of the present system.

Solutions with unstaggered $\phi_{n}$ and staggered $\psi_{n}$ components and two chemical potentials, $\lambda$ and $\mu$, are sought for as

$$
\phi_{n}(t)=e^{-i \lambda t} u_{n}, \quad \psi_{n}(t)=e^{-i \mu t}(-1)^{n} v_{n},
$$

where real $u_{n}$ and $v_{n}$ satisfy the following stationary equations,

$$
\begin{aligned}
(\lambda-1) u_{n}+\frac{1}{2}\left(u_{n+1}+u_{n-1}\right)+\left(u_{n}^{2}+\beta v_{n}^{2}\right) u_{n} & =0, \\
\left(\mu-\frac{1}{m}\right) v_{n}-\frac{1}{2 m}\left(v_{n+1}+v_{n-1}\right)+\left(v_{n}^{2}+\beta u_{n}^{2}\right) v_{n} & =0,
\end{aligned}
$$

that can be derived from the Lagrangian,

$$
\begin{aligned}
L= & \frac{1}{2} \sum_{n=-\infty}^{+\infty}\left[-\frac{1}{2}\left(u_{n+1}-u_{n}\right)^{2}+\lambda u_{n}^{2}+\frac{1}{2 m}\left(v_{n+1}-v_{n}\right)^{2}\right. \\
& \left.+\left(\mu-\frac{2}{m}\right) v_{n}^{2}+\frac{1}{2} u_{n}^{4}+\frac{1}{2} v_{n}^{4}+\beta u_{n}^{2} v_{n}^{2}\right]
\end{aligned}
$$

In the large- $m$ limit, which is tantamount to the TFA [9] for discrete equation ( $3 \mathrm{~b}$ ), this equation demonstrates that $v_{n}$ can be eliminated in favor of $u_{n}$; hence in this case the coupled stationary system reduces to a single equation.
In the next section, we present variational solutions based on an exponential ansatz for fundamental (single-peak) solitons, and continue the analysis in Sec. III by means of numerical methods. For given $\beta$ and $m$, we determine regions in the $(\lambda, \mu)$ plane in which single-peak numerical solutions exist and are stable. It is also found that the related energy surfaces, i.e., norms of the two components as functions of $\lambda$ and $\mu$, always decrease in $\lambda$ and either increase or decrease monotonically in $\mu$, depending on the sign of $1-\beta^{2}$. In this way, the generalized VK stability criterion for two-component solitary waves can be applied here $[15,16]$. In related two-component continuous systems [17-20], modeled by coupled continual NLS equations, one can introduce a new parameter (the ratio of $\lambda$ and $\mu$ ) and rescale the variables, to make the stationary states depend on one (rather than two) effective chemical potential [20]. Moreover, a generalized VK stability criteria was developed for a system of $N$ incoherently coupled continuous NLS equations in Ref. [21].

As for discrete systems, the single DNLS equation with the arbitrary power-law nonlinearity was studied, by means of the VA, in Ref. [22], and the stability of multisoliton bound states in the DNLS equation with the cubic self-focusing nonlinearity was investigated in Ref. [23]. A complex version of the VA made it later possible to make predictions about collisions between moving lattice solitons in the same basic model [24]. Another variational ansatz, relevant for DNLS solitons located on or anywhere between lattice cites, was elaborated in Ref. [25]. The VA was further generalized for the DNLS equation with the cubic-quintic on-site nonlinearity [26]. Very recently, the accuracy of the VA-based description of static discrete solitons and their stability, based on Ansätze with different numbers of free parameters, was investigated in a rigorous form in Ref. [27]. As concerns discrete two-component systems, the VA was used for studying the spontaneous symmetry breaking in parallel DNLS lattices, linearly coupled at all sites [28], or at a single site [29].

\section{ANALYTICAL APPROXIMATIONS}

\section{A. The variational approximation for the discrete solitons}

To apply the VA to the solution of Eqs. (3), we employ the exponential ansatz that was earlier used in the framework of other models [22,25-29]:

$$
u_{n}=A e^{-p|n|}, \quad v_{n}=B e^{-q|n|} .
$$

We find the decay rates of the wave forms in Eq. (5), $p$ and $q$, not from the variational principle, but by requiring the ansatz to satisfy the linearized limit of Eqs. (3) at $n \rightarrow \pm \infty$ :

$$
\begin{aligned}
& p=\ln (1-\lambda+\sqrt{-\lambda(2-\lambda)}), \\
& q=\ln (m \mu-1+\sqrt{m \mu(m \mu-2)}) .
\end{aligned}
$$

For $p$ and $q$ to be real and positive, the allowed ranges of chemical potentials $\mu$ and $\lambda$ are

$$
\lambda<0, \quad \mu-\frac{2}{m}>0 .
$$


Substituting ansatz (5) into Lagrangian (4) and carrying out the summation yields the effective Lagrangian,

$$
\begin{aligned}
2 L_{\mathrm{eff}}= & -A^{2} \tanh \left(\frac{p}{2}\right)+\frac{B^{2}}{m} \tanh \left(\frac{q}{2}\right)+\lambda A^{2} \operatorname{coth} p \\
& +\left(\mu-\frac{2}{m}\right) B^{2} \operatorname{coth} q+\frac{A^{4}}{2} \operatorname{coth}(2 p) \\
& +\frac{B^{4}}{2} \operatorname{coth}(2 q)+\beta A^{2} B^{2} \operatorname{coth}(p+q),
\end{aligned}
$$

which gives rise to the variational equations, $\partial L_{\text {eff }} / \partial\left(A^{2}\right)=$ $\partial L_{\text {eff }} / \partial\left(B^{2}\right)=0$, i.e.,

$$
A^{2} \operatorname{coth}(2 p)+[\beta \operatorname{coth}(p+q)] B^{2}=\tanh \left(\frac{p}{2}\right)-\lambda \operatorname{coth} p,
$$

$$
\begin{aligned}
& {[\beta \operatorname{coth}(p+q)] A^{2}+B^{2} \operatorname{coth}(2 q)} \\
& \quad=-\frac{1}{m} \tanh \left(\frac{q}{2}\right)-\left(\mu-\frac{2}{m}\right) \operatorname{coth} q .
\end{aligned}
$$

As seen from Eqs. (7) and (9b), solutions to the variational equations with positive $A^{2}$ and $B^{2}$ do not exist in the case of $\beta>0$, but a solution may exist at $\beta<0$.

The fact that the fundamental solitons of the mixed unstaggered-staggered type may exist as the bound state of two components, which in isolation support solely ordinary unstaggered solitons (through the self-attractive SPM), suggests identifying the solitons of the mixed type as symbiotic ones, cf. Ref. [30], where symbiotic solitons were defined in the opposite case, for the continual system with the self-repulsive SPM and attractive XPM nonlinearities. On the other hand, the staggering effectively reverses the signs of the SPM nonlinearity and external potential; therefore, in the presence of a large-amplitude unstaggered component, the staggered one may be considered as a soliton with the intrinsic self-repulsive nonlinearity, trapped in the attractive external potential. Such a mode tends to exist and be stable, unless the effective intrinsic self-repulsion is too strong, making the existence of the trapped mode impossible [31].

We also note (this remark will be relevant for comparison with some numerical results presented in the next section) that a solution to Eqs. (9), considered as a linear system for $A^{2}$ and $B^{2}$, may not exist when the determinant of the system vanishes, i.e.,

$$
\operatorname{coth}(2 p) \operatorname{coth}(2 q)-\beta^{2} \operatorname{coth}^{2}(p+q)=0 .
$$

Nevertheless, a solution is possible under condition (10) if the right-hand sides of Eqs. (9) are related in the same way as the two rows of the degenerate determinant, i.e.,

$$
\begin{aligned}
\tanh \left(\frac{p}{2}\right)-\lambda \operatorname{coth} p= & -\frac{\operatorname{coth}(2 p)}{\beta \operatorname{coth}(p+q)}\left[\frac{1}{m} \tanh \left(\frac{q}{2}\right)\right. \\
& \left.+\left(\mu-\frac{2}{m}\right) \operatorname{coth} q\right] .
\end{aligned}
$$

\section{B. Three-layer solitons for $1 / m \rightarrow 0$ (the discrete Thomas-Fermi approximation)}

There is another case in which we can determine properties of the solution in an analytical form. When the staggered species is very heavy, i.e., $m \rightarrow \infty$ in Eq. (1b), the second equation from system (3), at lowest order, takes the local form:

$$
\left[\mu+\left(v_{n}^{2}+\beta u_{n}^{2}\right)\right] v_{n}=0 .
$$

Equation (12) has three possible solutions, viz.,

$$
v_{n}^{2}=-\mu-\beta u_{n}^{2},
$$

or $v_{n}=0$, which may be used to eliminate $v_{n}$ in favor of $u_{n}$; cf. a similar approach allowing one to eliminate a heavy-fermionic component in a Bose-Fermi mixture [32]. Accordingly, discrete solitons, composed of three layers, can be built as follows: In the central region (inner layer), we use relation (13) and substitute it into the first equation of system (3), which yields

$$
[(\lambda-1)-\beta \mu] u_{n}+\frac{1}{2}\left(u_{n+1}+u_{n-1}\right)+\left(1-\beta^{2}\right) u_{n}^{3}=0,
$$

i.e., the stationary DNLS equation which gives rise to soliton solutions. Requiring this solution, in the central region, to be a part of a discrete soliton with a single peak and centered at $n=0$, then one must have $\beta^{2}<1$ and $\lambda<\beta \mu$.

It follows from Eq. (13) that, since $\mu>0$ [see Eq. (7)], for $v_{n}^{2}$ to be positive, one must take $-1<\beta<0$, which yields $v_{n}^{2}=(-\beta)\left(u_{n}^{2}-U^{2}\right)$, with $U^{2} \equiv-\mu / \beta>0$. Provided that $u_{0}^{2}>U^{2}$, one thus has $v_{n}^{2}>0$ at $n=0$ and in some region around $n=0$ (in the inner layer of the solution, as defined above). However, the positiveness of the so defined $v_{n}^{2}$ will be lost at $|n|>N$ with $N$ large enough, as $u_{n}^{2}$ for soliton solutions decays at $n \rightarrow \infty$. Thus, for $n>N$ and $n<-N$ (in the two outer layers), the discrete mode can be extended upon taking the other root of Eq. (12) for $v_{n}$, namely, $v_{n} \equiv 0$, which thus causes $u_{n}$ to satisfy the usual DNLS equation, following from Eq. (3b) with $v_{n} \equiv 0$ :

$$
(\lambda-1) u_{n}+\frac{1}{2}\left(u_{n+1}+u_{n-1}\right)+u_{n}^{3}=0 .
$$

Obviously, Eq. (15) has the usual solution vanishing as $|n| \rightarrow \infty$ for $\lambda<0$ [recall $\lambda<0$ is imposed by Eq. (7)]; thus the composite soliton can be constructed by combining the appropriate solutions in the inner and outer layers. The conditions of matching the discrete fields at $n= \pm N$, which includes setting $v_{N}=0$ (as required by the TFA), imposes two constraints on the set of parameters $\lambda, \mu$, and $N$; hence the solution is expected to exist along a curve in the plane of $(\lambda, \mu)$, which is corroborated by numerical findings presented in the next section. Note that in the framework of the present approximation, there is actually no difference between the unstaggered and staggered forms of the solution for $v_{n}$, as only $v_{n}^{2}$ is determined by Eq. (13).

\section{NUMERICAL SOLITON SOLUTIONS}

\section{A. The formulation of the numerical problem}

We look for numerical solutions to Eqs. (93) for spatially symmetric solitons, with $u_{-n}=u_{+n}, v_{-n}=v_{+n}$, and both fields $u_{n}$ and $v_{n}$ monotonously decaying with the increase of $n$, but never changing their signs, to support the unstaggered and staggered shapes of the underlying components $\phi_{n}$ and $\psi_{n}$, 


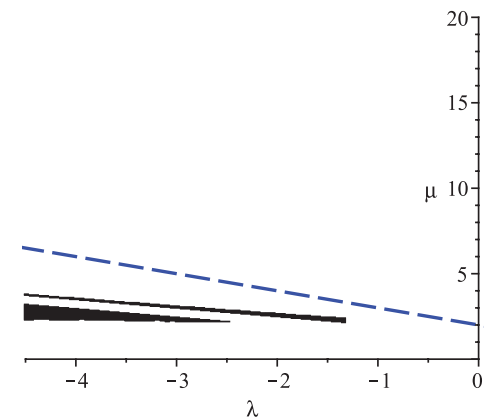

(a)

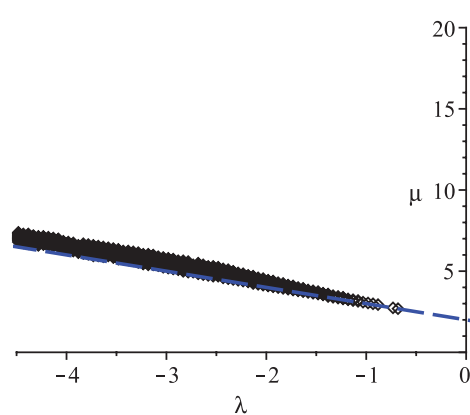

(b)

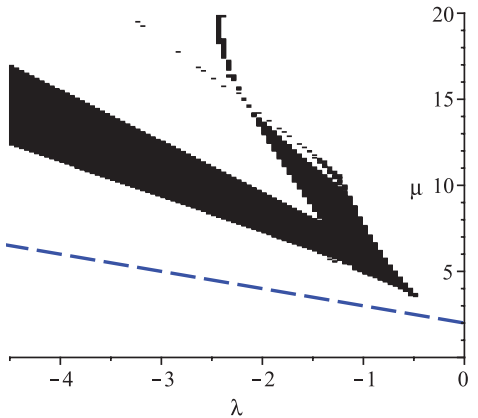

(c)

FIG. 1. (Color online) Existence regions (black) for the numerically found single-peak discrete solitons in the $(\lambda, \mu)$ plane, for $m=1$ and (a) $\beta=-0.5$, (b) $\beta=-1.01$, (c) $\beta=-2$. The dashed blue line corresponds to $\mu=2-\lambda$ [see Eq. (19)], to which the existence region shrinks in the case of $\beta=-1$.

respectively, according to Eq. (2). At $n=0$, Eqs. (3) yield

$$
\begin{aligned}
& u_{1}=-\left[u_{0}^{2}+\beta v_{0}^{2}+(\lambda-1)\right] u_{0}, \\
& v_{1}=m\left[v_{0}^{2}+\beta u_{0}^{2}+\left(\mu-\frac{1}{m}\right)\right] v_{0} .
\end{aligned}
$$

According to the above conditions, solutions to Eqs. (16) must satisfy constraints $u_{0}>u_{1}>0$ and $v_{0}>v_{1}>0$, thereby implying that

$$
\begin{aligned}
& -1<u_{0}^{2}+\beta v_{0}^{2}+(\lambda-1)<0, \\
& \frac{1}{m}<v_{0}^{2}+\beta u_{0}^{2}+\mu<\frac{2}{m} .
\end{aligned}
$$

Continuing in this manner, i.e., imposing bounds $u_{1}>u_{2}>0$, $v_{1}>v_{2}>0$, and so on, as follows from Eqs. (3) at $n=$ $1,2, \ldots$, one successively restricts the region of the $(\lambda, \mu)$ plane in which the soliton solutions are possible.

The numerical solution of Eqs. (3) was carried out by means of a discrete version of the shooting method, which used the VA-predicted solution as the initial guess, and was iterated until discrete wave forms monotonously decreasing with $n$ without the change of the sign, up to the level of $\left(u_{n}, v_{n}\right) \sim$ $10^{-5}\left(u_{0}, v_{0}\right)$, were found.

For stability testing, we introduced initial perturbations, multiplying the stationary solutions by

$$
[1+\delta \exp (i k n)],
$$

with perturbation amplitude $\delta \simeq 0.05$ and $k N \sim 1$, where $N$ is the effective size of the discrete soliton. Then, the evolution of the thus perturbed solution was simulated forward in time until $t=50$. The results of the simulations were characterized by "stability numbers" $S_{\phi}$ and $S_{\psi}$ for the two components, which are defined as root-mean-square changes in the relative amplitude of the solution, compared to the initial values, over the part of the lattice where the discrete soliton is located. For stable solutions, we obtain $\left|S_{\phi}, S_{\psi}\right| \ll 1$, while for unstable ones $\left|S_{\phi}, S_{\psi}\right|$ grow to values $\gtrsim 1$.

Since these solitons are symbiotic, one might suspect that they could be unstable to efforts to pull their two components apart. We have also checked this possibility numerically, as above, by taking wave numbers $k_{u}$ and $k_{v}$ with opposite signs in perturbation factors (18) for the two fields. All solutions that we tested in this way, which had tested out to be stable against other perturbations, were found to be stable in this sense too.

\section{B. Dependence of solutions on the parameters}

In agreement with the prediction of the VA, numerical solutions for the solitons were found solely for $\beta<0$, and, as suggested by Eq. ((14)), $\beta=-1$ is a critical value. When $m=1$, we can demonstrate this with numerical results, which makes it possible to identify two distinct cases, $\beta<-1$ and $-1<\beta<0$, as seen in Fig. 1. When $\beta$ approaches -1 from either side, we find solutions in a region which shrinks toward the line

$$
\mu=2-\lambda
$$

in the $(\lambda, \mu)$ plane. It is worthy to note that, as can be found from inspection of Eqs. (9) and (11), both these equations reduce precisely to Eq. (19) in the case of $m=1$ and $\beta=-1$; i.e.,

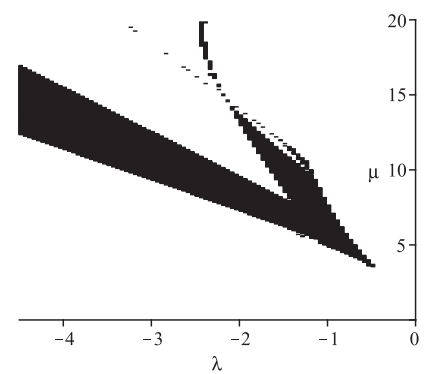

(a)

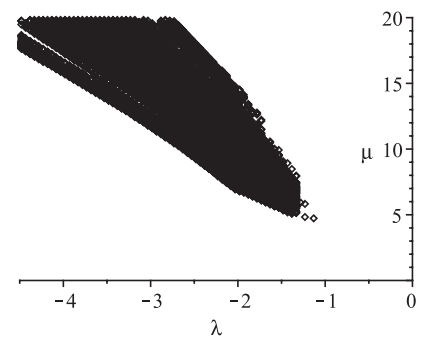

(c)

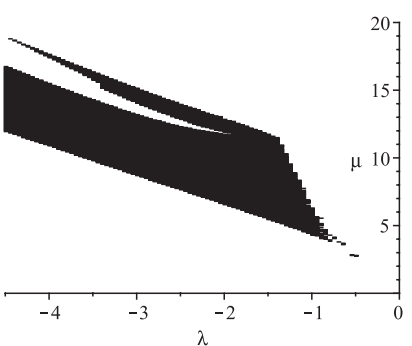

(b)

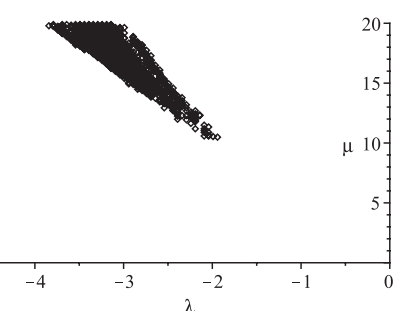

(d)
FIG. 2. The same as in Fig. 1 [except that the blue line (19) is not shown] for $\beta=-2$ and (a) $m=1$, (b) $m=2$, (c) $m=5$, (d) $m=10$. 


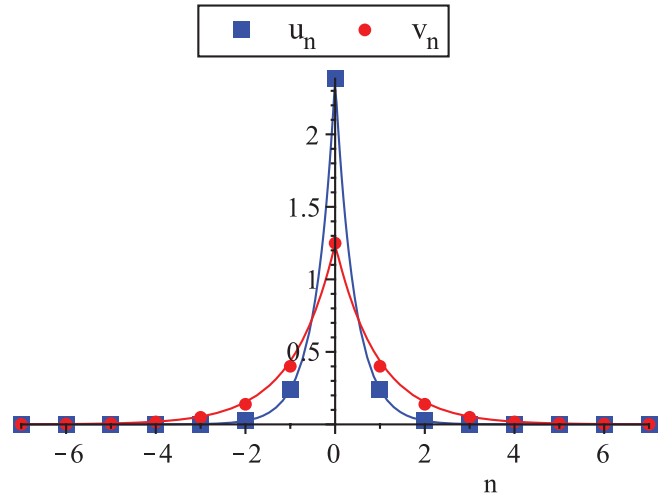

(a)

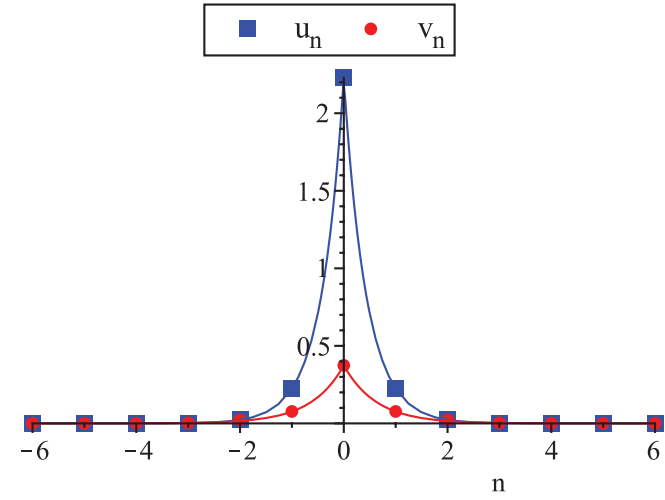

(b)

FIG. 3. (Color online) Examples of discrete solitons for $\beta=-0.5, m=1$, and $(\lambda, \mu)=(-4,2.6)$ (a) or $(\lambda, \mu)=(-4,3.55)$ (b). Symbols and lines depict the numerical solutions and prediction of the variational approximation, respectively. Both solitons shown here are stable.

only the "double-degenerate" solution selected by Eqs. (9) and (11) survives in this case. Note also that Eqs. (6) with $m=1$ yield equal decay rates $p$ and $q$ for the two components of the soliton exactly under the condition given by Eq. (19); i.e., the soliton surviving in the limit of $m=1$ and $\beta=-1$ is characterized by equal localization lengths of the two components.
Moving away from the critical value, $\beta=-1$, in either direction, Fig. 1 shows that the existence region of the numerically found solitons widens, and, simultaneously, the region moves away from line (19), staying on one side of this line, depending on the sign of $1+\beta$. For $\beta$ sufficiently far from -1 , additional solution regions begin to split off from

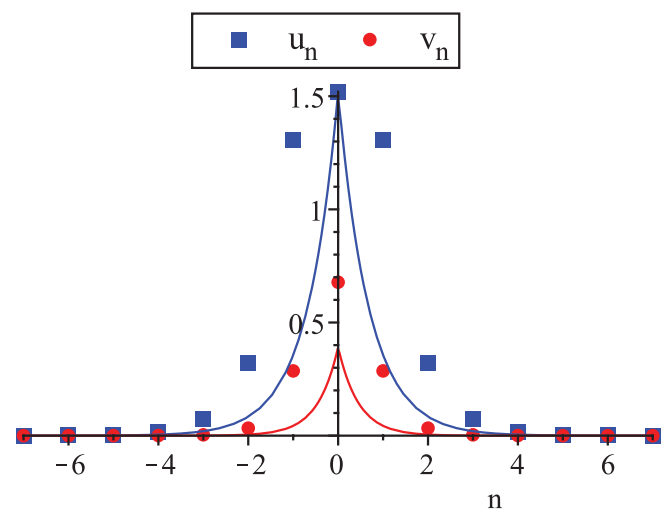

(a)

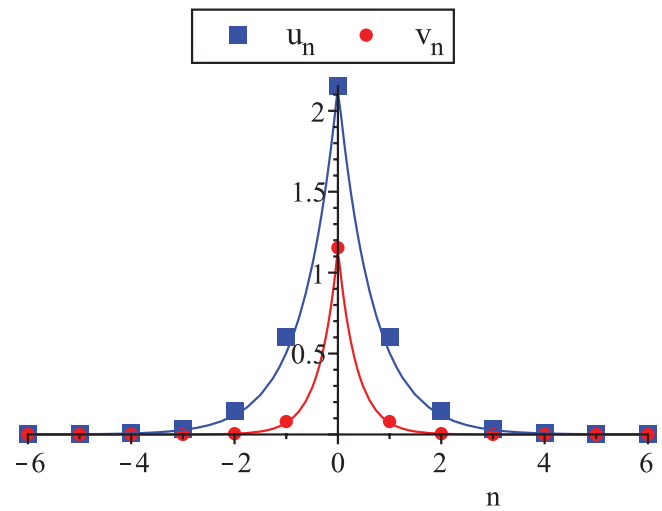

(c)

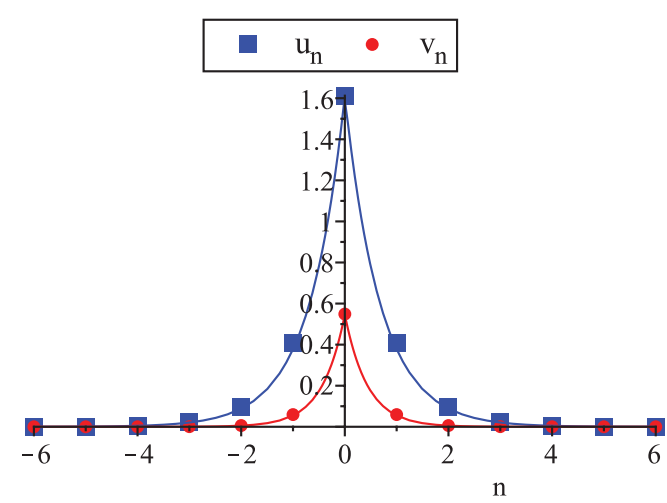

(b)

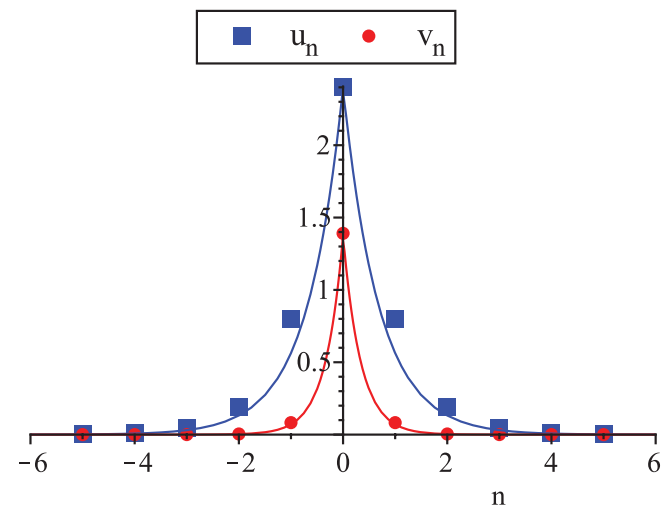

(d)

FIG. 4. (Color online) The same as in Fig. 3 , for $\beta=-2, m=1$, and $(\lambda, \mu)=(-1.25,5.58)(\mathrm{a}),(\lambda, \mu)=(-1.25,6.005)(\mathrm{b}),(\lambda, \mu)=$ $(-1.25,9)(\mathrm{c})$, and $(\lambda, \mu)=(-1.25,10.7)(\mathrm{d})$. For the soliton in panel (a), the variational approximation provides a poor fit to the numerical solution, and this soliton is unstable. Other solitons are well approximated by the variational ansatz and are stable. 


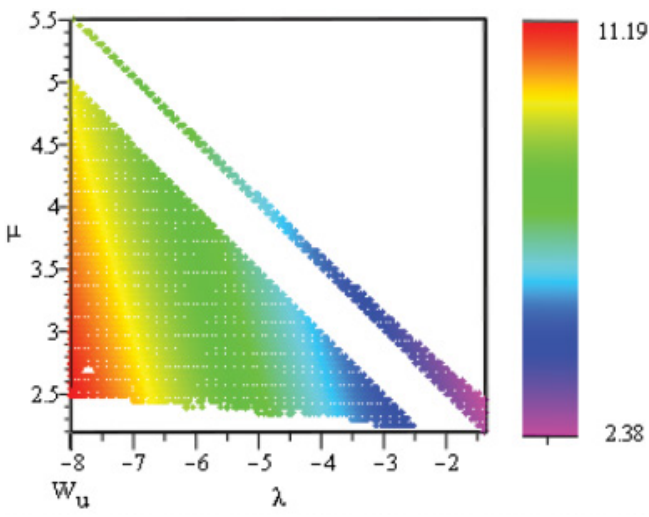

(a)

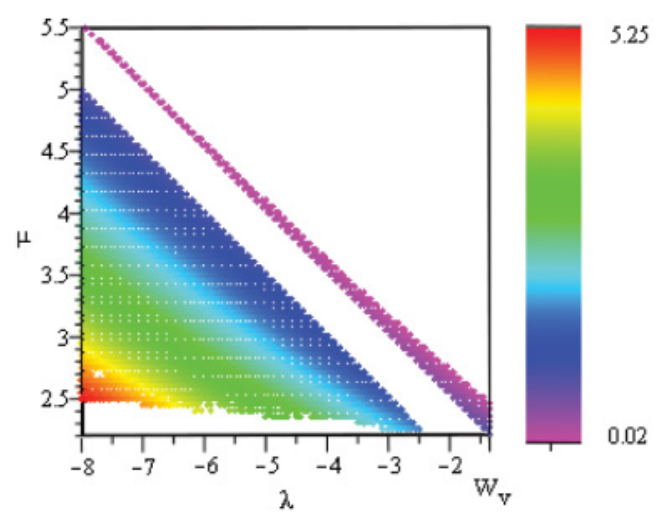

(b)

FIG. 5. (Color online) Color-coded plots of the energy surfaces $W_{u}(\lambda, \mu)$ (a) and $W_{v}(\lambda, \mu)$ (b) in the existence regions for the discrete solitons in the $(\lambda, \mu)$ plane, at $m=1$ and $\beta=-0.5$. The energy increases with $\lambda$ (holding $\mu$ fixed) and decreases with $\mu$ (holding $\lambda$ fixed).

the primary one in the $(\lambda, \mu)$ plane. These additional solution branches break off from the primary "trunk" at small $|\lambda|$, then shrinking and disappearing as $|\lambda|$ increases, while the main trunk widens as $\lambda \rightarrow-\infty$.

With the increase of the relative-mass parameter $m$, the existence region of the soliton solutions in the $(\lambda, \mu)$ plane shrinks, following Eqs. (17). This trend is observed in Fig. 2, which suggests that the region contracts toward a line in the $(\lambda, \mu)$ plane at $m \rightarrow \infty$ (as predicted by the TFA presented above). In the case when $\beta$ is far enough from -1 to permit additional branches in the existence diagrams, we observe that such branches collapse into the primary one (the "trunk"), which then itself collapses into a line, as can be seen in Fig. 2 for $\beta=-2$. It is also worthy to note that the bottom boundary of the existence region in Fig. 2 moves upward with the increase of $m$ at fixed $\lambda$.

Examples of the solitons, including the juxtaposition of their numerically found and VA-predicted profiles, are displayed in Fig. 3 for $\beta=-0.5$ and $m=1$. Fixing $\lambda=-4$, we pick solutions from the larger lower stability region and the upper one in Fig. 1(a) corresponding to $\mu=2.6$ and $\mu=3.55$, respectively. Note that, while the profiles of the unstaggered component $u_{n}$ are very similar to one another, the solutions for $v_{n}$ are different. In both cases, the variational solutions agree well with their numerical counterparts.

In Fig. 4, the solitons are plotted for the four branches of the existence region in Fig. 1(c) when $\beta=-2$ and $m=1$. The first solution, shown in Fig. 4(a), belongs to a very narrow existence branch, which is barely discernible in Fig. 1(c) (its vertical width is $\Delta \mu<0.002$ ), and exists along the bottom right of the main existence region (near the edge where $\lambda \approx-1.3$ and $\mu \approx 5.0$ ). The other solutions are taken from the large lower existence region [Fig. 4(b)], the large upper one [Fig. 4(c)], and the thin upper stripe which splits off from the large upper branch [Fig. 4(d)]. Solutions from the lowest region [Fig. 4(a)] feature wider profiles in $u_{n}$ (note that both $u_{ \pm 1}$ for them are on the same order of magnitude as $u_{0}$ ) than do the solutions from all the other branches, which exhibit sharp profiles and agree well with the VA. On the contrary, the broad profile for $u_{n}$ in Fig. 4(a) cannot be approximated properly by the exponential ansatz (5).

Because the numerical method employed here starts in a region where the variational equations, Eqs. (9), have a solution, we cannot be absolutely sure that numerical solutions exist only in the dark areas shown in Figs. 1 and 2. In principle, other branches of numerical solutions might exist too, being unrelated to the VA, although this does not seem plausible.

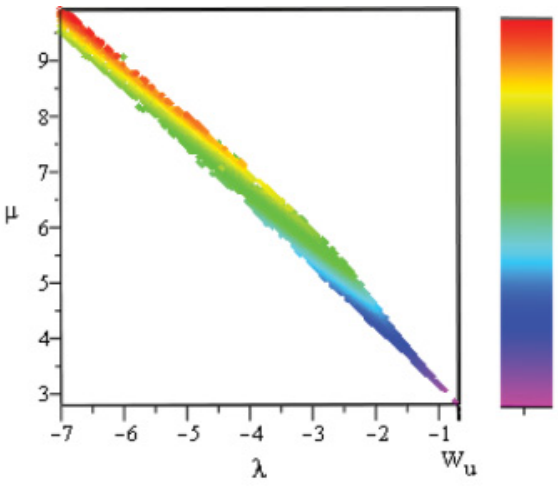

(a)
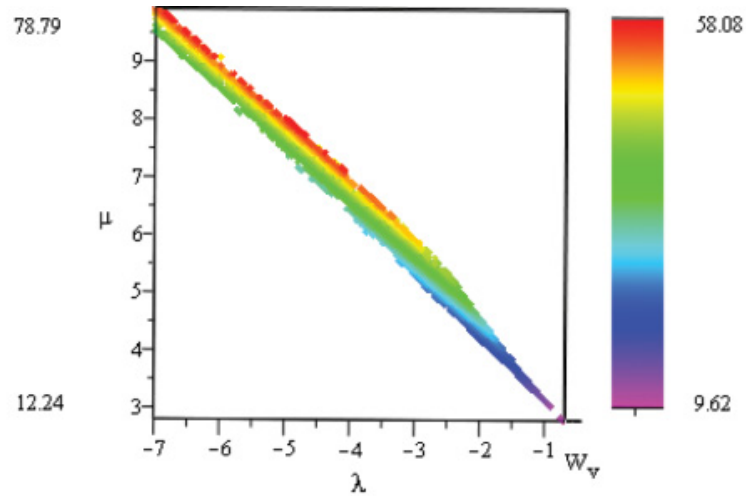

(b)

FIG. 6. (Color online) The same as in Fig. 5, but for $m=1$ and $\beta=-1.01$. The energy surface decreases with $\lambda$ (holding $\mu$ fixed) and increases with $\mu$ (holding $\lambda$ fixed). 


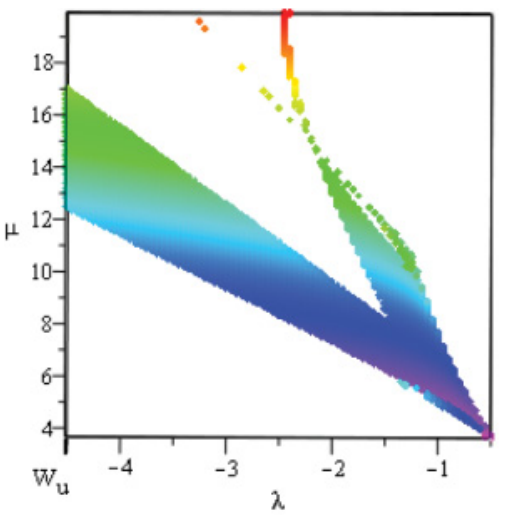

(a)
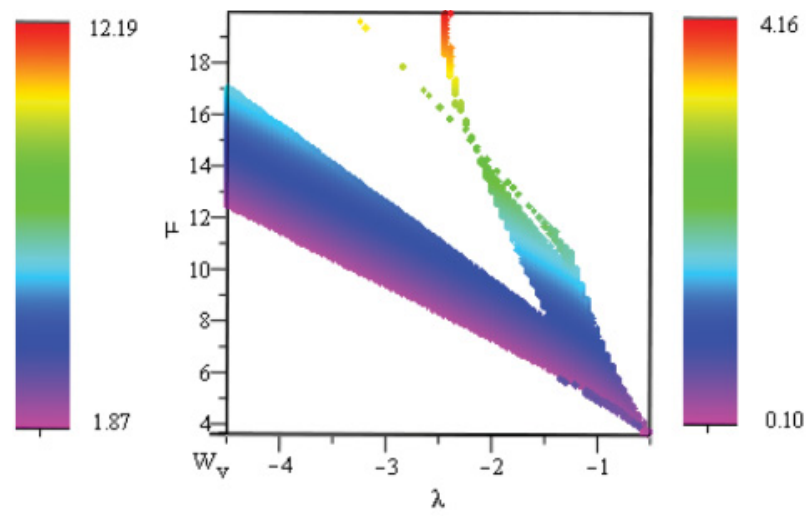

(b)

FIG. 7. (Color online) The same as in Figs. 5 and 6, but for $m=1$ and $\beta=-2$. The energy decreases with $\lambda$ (holding $\mu$ fixed) and increases with $\mu$ (holding $\lambda$ fixed).

\section{Soliton stability}

Systematic simulations of the evolution of perturbed solitons shown in Figs. 3 and 4 confirm that they are stable, with the exception of the one in Fig. 4(a). Further, systematic tests clearly suggest that the numerically found solitons are stable if their shapes are close to those predicted by the VA, whereas "broad" solutions, which disagree with the VA, turn out to be unstable. Actually, such unstable solitons are found only near the lower boundary of the regions shown in Fig. 1.

In order to deduce the stability in a more general way, we define the energies (norms) of the components,

$$
W_{u}(\lambda, \mu)=\sum_{n=-\infty}^{\infty}\left|u_{n}\right|^{2}, \quad W_{v}(\lambda, \mu)=\sum_{n=-\infty}^{\infty}\left|v_{n}\right|^{2}
$$

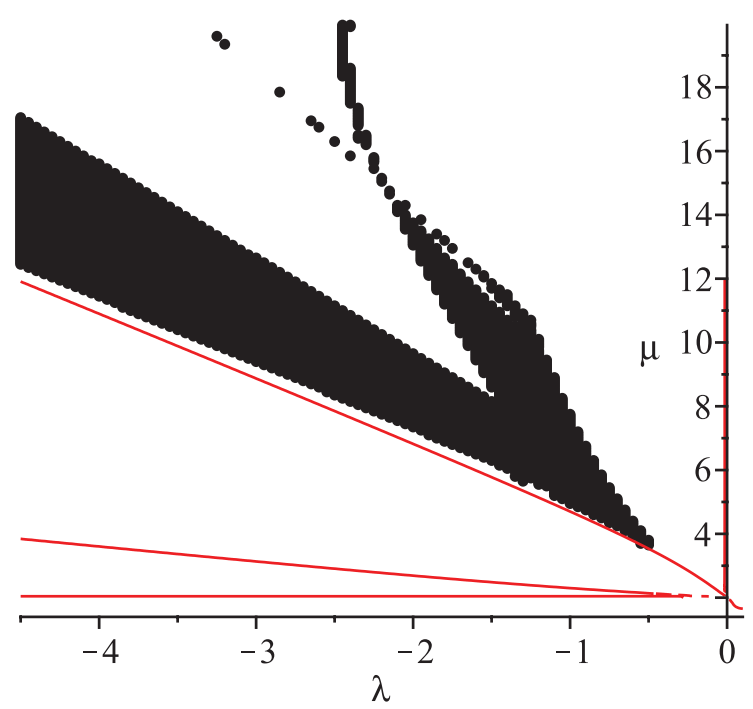

FIG. 8. (Color online) The numerically found existence regions (black) in the $(\lambda, \mu)$ plane for the single-peak solitons, juxtaposed with the (red online, dark gray in print) curves defined by $\partial\left(W_{u}, W_{v}\right) / \partial(\lambda, \mu)=0$, as produced by the variational approximation, for $m=1$ and $\beta=-2$. The solitons existing above the red line are stable.
In Fig. $5 W_{u}(\lambda, \mu)$ and $W_{v}(\lambda, \mu)$ are plotted for $m=1$ and $\beta=0.5$, and in Figs. 6 and 7 we do the same for $\beta=-1.1$ and $\beta=-2$, respectively. There is a noticeable difference between the energy surfaces for the $\beta<-1$ and $-1<\beta<0$ cases. In all cases considered, the energy surfaces are monotonous in $\lambda$ and $\mu$ over the stability regions (this finding agrees with the stability results reported in Ref. [22]). However, for $-1<\beta<$ 0 , the energy increases with $\lambda$ (holding $\mu$ fixed) and decreases with $\mu$ (holding $\lambda$ fixed), as seen in Fig. 5 . The opposite feature is observed at $\beta<-1$ : The energy decreases with $\lambda$ at fixed $\mu$ and increase in $\mu$ at fixed $\lambda$.

More can be stated about the stability by means of the VA. The substitution of ansatz (5) into Eqs. (20) yields

$$
\begin{aligned}
& W_{u}(\lambda, \mu)=A \sum_{n=-\infty}^{\infty} e^{-2 p|n|}=A \operatorname{coth}(p), \\
& W_{v}(\lambda, \mu)=B \sum_{n=-\infty}^{\infty} e^{-2 q|n|}=B \operatorname{coth}(q),
\end{aligned}
$$

where $A$ and $B$ are functions of $\lambda$ and $\mu$ determined by Eqs. (9). As is known from the generalized VK criterion for systems with two conserved norms [16], a stability change

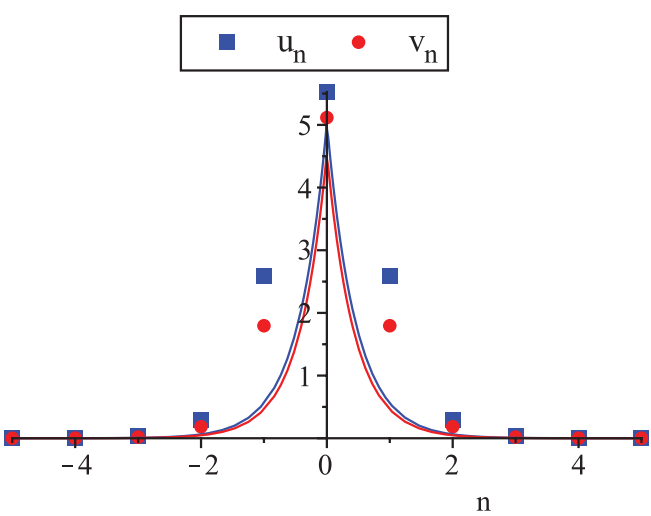

FIG. 9. (Color online) An example of a discrete soliton for $\beta=$ $-1.01, m=1$, and $(\lambda, \mu)=(-3.55,6)$. Symbols and lines depict the numerical solutions and prediction of the variational approximation, respectively. The soliton shown here is unstable. 


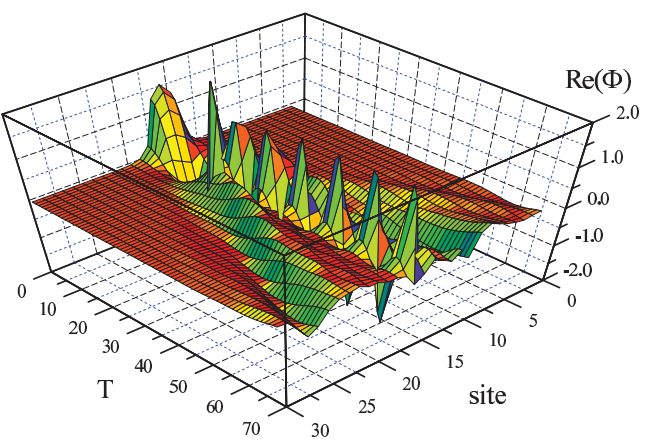

(a)

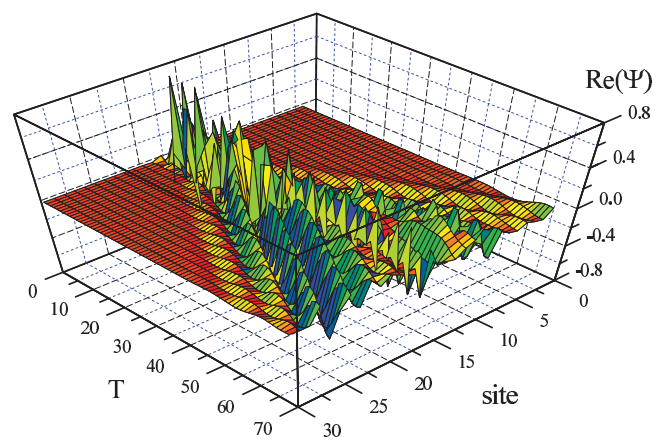

(b)

FIG. 10. (Color online) Plots of (a) $\phi_{n}(t)$ and (b) $\psi_{n}(t)$ for the evolution of the unstable soliton from Fig. 4(a), which corresponds to $\beta=-2, m=1,(\lambda, \mu)=(-1.25,5.58)$. Note the escaping radiation in both components, and the oscillating breathers which are left behind.

occurs when Jacobian $\partial\left(W_{u}, W_{v}\right) / \partial(\lambda, \mu)$ changes its sign. We follow this approach in Fig. 8, where the zero locus of the Jacobian is plotted, along with the region of the existence of the numerically found solitons, for $\beta=-2$ and $m=1$. It is observed that the stability change predicted by the VA nearly coincides with the lower boundary of the existence region. The agreement is not perfect since the VA does not produce exact results, but the mismatch is quite small. The majority of the soliton solutions, which are located above the stability-change locus, are stable; unstable are the solitons, such as the broad one displayed in Fig. 4(a), which are found in a tiny area adjacent to the lower boundary which is actually bounded by the Jacobian's zero locus crossing the existence region.

Comparing Figs. 5-7, we see that as $\beta \rightarrow-1$, the energy values for the obtained solitons increase. We observe that this, in turn, corresponds to a change in the stability, and it was found that solitons occurring in the narrow region shown in Fig. 1(b) for $\beta=-1.01$ are unstable. One such soliton is plotted in Fig. 9. Notice that the unstable numerical solution is again essentially wider than its variational counterpart; i.e., as in Fig. 4(a), the variational approximation is a poor fit to the broad soliton. We have tested the stability of similar soliton solutions for $\beta=-1.10$ and $\mu=6.0$, and have found them to be stable. So the instability region appears to be localized around $\beta=-1$. Lastly, direct simulations demonstrate that the unstable broad soliton solutions, such as the one displayed in Fig. 4(a), decay into a combination of multiple breathers and emitted radiation, as seen in the example in Fig. 10.

\section{CONCLUSIONS}

We have introduced the symmetric system of DNLS (discrete nonlinear Schrödinger) equations with the self-attractive on-site SPM nonlinearity and repulsive XPM interaction, which supports two-component solitons of the symbiotic unstaggered-staggered type. The system may be implemented in a mixture of two BEC species with identical or different atomic masses, and (in principle) in arrays of bimodal optical waveguides. In the analytical part of the work, the VA (variational approximation) was developed, based on the exponential ansatz for the fundamental (single-peak) solitons. In the limit of the large relative mass of the two species, the TFA was elaborated too, which reduces the coupled system to two different single-component DNLS equations in the inner and outer layers of the solutions. Further, by means of the numerical solution we have identified areas in the plane of the two chemical potentials (propagation constants) where discrete solitons exist. It has been inferred that the VA and TFA agree well with the numerical solutions, except for a stripe near the lower existence boundary, where broad solitons are poorly approximated by the exponential ansatz. Direct simulations of the evolution of the perturbed solitons demonstrate that all the solitons which are well approximated by the VA (i.e., almost all the solutions) are stable. Only the broad solitons, which are not accommodated by the VA, are unstable. The results for the stability can be accurately predicted by means of the generalized VK criterion for the two-component system (with the stability change corresponding to the vanishing of the respective Jacobian), realized in terms of the VA.

It may be interesting to extend the work by considering multisoliton (multipeak) bound states of the unstaggeredstaggered type. A challenging problem is to generalize the system for two-dimensional lattices and various types of discrete two-dimensional solitons, including solitary vortices.

\section{ACKNOWLEDGMENTS}

B.A.M. appreciates partial support from the Binational (US-Israel) Science Foundation, through Grant No. 2010239. R.A.V. was supported in part by a National Science Foundation fellowship.
[1] P. G. Kevrekidis, Discrete Nonlinear Schrödinger Equation: Mathematical Analysis, Numerical Computations, and Physical Perspectives (Springer, Berlin, 2009).
[2] D. N. Christodoulides and R. I. Joseph, Opt. Lett. 13, 794 (1988).

[3] H. S. Eisenberg, Y. Silberberg, R. Morandotti, A. R. Boyd, and J. S. Aitchison, Phys. Rev. Lett. 81, 3383 (1998). 
[4] J. W. Fleischer, G. Bartal, O. Cohen, T. Schwartz, O. Manela, B. Freedman, M. Segev, H. Buljan, and N. K. Efremidis, Opt. Express 13, 1780 (2005).

[5] A. Szameit, D. Blömer, J. Burghoff, T. Schreiber, T. Pertsch, S. Nolte, and A. Tünnermann, Opt. Express 13, 10552 (2005); A. Szameit, J. Burghoff, T. Pertsch, S. Nolte, A. Tünnermann, and F. Lederer, ibid. 14, 6055 (2006).

[6] F. Lederer, G. I. Stegeman, D. N. Christodoulides, G. Assanto, M. Segev, and Y. Silberberg, Phys. Rep. 463, 1 (2008).

[7] F. S. Cataliotti, S. Burger, C. Fort, P. Maddaloni, F. Minardi, A. Trombettoni, A. Smerzi, and M. Inguscio, Science 293, 843 (2001); M. Greiner, O. Mandel, T. Esslinger, T. W. Hänsch, and I. Bloch, Nature (London) 415, 39 (2002).

[8] A. Trombettoni and A. Smerzi, Phys. Rev. Lett. 86, 2353 (2001); G. L. Alfimov, P. G. Kevrekidis, V. V. Konotop, and M. Salerno, Phys. Rev. E 66, 046608 (2002); R. Carretero-Gonzalez and K. Promislow, Phys. Rev. A 66, 033610 (2002); N. K. Efremidis and D. N. Christodoulides, ibid. 67, 063608 (2003); M. A. Porter, R. Carretero-González, P. G. Kevrekidis, and B. A. Malomed, Chaos 15, 015115 (2005).

[9] L. Pitaevskii and S. Stringari, Bose-Einstein Condensation (Clarendon Press, Oxford, 2003).

[10] D. E. Pelinovsky, Localization in Periodic Potentials: From Schrödinger Operators to the Gross-Pitaevskii Equation (Cambridge University Press, Cambridge, 2011).

[11] Y. S. Kivshar and M. I. Molina, Wave Motion 45, 59 (2007); D. Mihalache, D. Mazilu, F. Lederer, and Y. S. Kivshar, Opt. Lett. 32, 2091 (2007).

[12] S. K. Adhikari and B. A. Malomed, Phys. Rev. A 77, 023607 (2008).

[13] A. Gubeskys, B. A. Malomed, and I. M. Merhasin, Phys. Rev. A 73, 023607 (2006).

[14] M. Zaccanti, C. D’Errico, F. Ferlaino, G. Roati, M. Inguscio, and G. Modugno, Phys. Rev. A 74, 041605(R) (2006); S. B. Papp, J. M. Pino, and C. E. Wieman, Phys. Rev. Lett. 101, 040402 (2008); S. E. Pollack, D. Dries, M. Junker, Y. P. Chen, T. A. Corcovilos, and R. G. Hulet, ibid. 102, 090402 (2009); P. Zhang, P. Naidon, and M. Ueda, ibid. 103, 133202 (2009).

[15] N. G. Vakhitov and A. A. Kolokolov, Radiophys. Quantum Electron. 16, 783 (1973).
[16] L. Bergé, Phys. Rep. 303, 259 (1998); E. A. Kuznetsov and F. Dias, ibid. 507, 43 (2011).

[17] Y. Chen and J. Atai, Opt. Commun. 150, 381 (1998).

[18] E. A. Ostrovskaya, Y. S. Kivshar, D. V. Skryabin, and W. J. Firth, Phys. Rev. Lett. 83, 296 (1999).

[19] A. I. Yakimenko, V. M. Lashkin, and O. O. Prikhodko, Phys. Rev. E 73, 066605 (2006).

[20] A. I. Yakimenko, O. O. Prikhodko, and S. I. Vilchynskyi, Phys. Rev. E 82, 016605 (2010).

[21] D. E. Pelinovsky and Y. S. Kivshar, Phys. Rev. E 62, 8668 (2000).

[22] B. Malomed and M. Weinstein, Phys. Lett. A 220, 91 (1996).

[23] T. Kapitula, P. G. Kevrekidis, and B. A. Malomed, Phys. Rev. E 63, 036604 (2001); P. G. Kevrekidis, B. A. Malomed, and A. R. Bishop, J. Phys. A: Math. Gen. 34, 9615 (2001).

[24] I. E. Papacharalampous, P. G. Kevrekidis, B. A. Malomed, and D. J. Frantzeskakis, Phys. Rev. E 68, 046604 (2003).

[25] D. J. Kaup, Math. Comput. Simul. 69, 322 (2005).

[26] R. Carretero-González, J. D. Talley, C. Chong, and B. A. Malomed, Physica D 216, 77 (2006); C. Chong, R. CarreteroGonzález, B. A. Malomed, and P. G. Kevrekidis, ibid. 238, 126 (2009).

[27] C. Chong, D. E. Pelinovsky, and G. Schneider, Physica D 241, 115 (2011).

[28] G. Herring, P. G. Kevrekidis, B. A. Malomed, R. CarreteroGonzález, and D. J. Frantzeskakis, Phys. Rev. E 76, 066606 (2007).

[29] Lj. Hadžievski, G. Gligorić, A. Maluckov, and B. A. Malomed, Phys. Rev. A 82, 033806 (2010); M. D. Petrović, G. Gligorić, A. Maluckov, Lj. Hadžievski, and B. A. Malomed, Phys. Rev. E 84, 026602 (2011); M. Stojanović, A. Maluckov, Lj. Hadzievski, and B. A. Malomed, Physica D 240, 1489 (2011).

[30] V. M. Pérez-García and J. B. Beitia, Phys. Rev. A 72, 033620 (2005); S. K. Adhikari, Phys. Lett. A 346, 179 (2005); Phys. Rev. A 72, 053608 (2005); J. Phys. A 40, 2673 (2007).

[31] L. D. Carr, M. J. Holland, and B. A. Malomed, J. Phys. B 38, 3217 (2005).

[32] S. K. Adhikari, B. A. Malomed, L. Salasnich, and F. Toigo, Phys. Rev. A 81, 053630 (2010). 